\title{
Melanoma Detection Based on Mahalanobis Distance Learning and Constrained Graph Regularized Nonnegative Matrix Factorization
}

\author{
Yanyang Gu, Jun Zhou \\ School of Information and Communication Technology \\ Griffith University, Nathan, Australia \\ yanyang.guegriffithuni.edu.au, jun.zhou@griffith.edu.au \\ Bin Qian \\ School of Computer Science and Engineering \\ Nanjing University of Science and Technology, Nanjing, China \\ 311062198@njust. edu.cn
}

\begin{abstract}
Melanoma is the most fatal form of all skin cancer types. An early screening of melanoma can greatly contribute to successful treatment, hence reliable early detection systems are highly demanded. In this paper, we propose a novel melanoma detection method based on Mahalanobis distance learning and constrained graph regularized nonnegative matrix factorization. The proposed method allows supervised learning for feature dimensionality reduction by incorporating both global geometry and local manifold, so as to enhance the discriminability of the classification performance. The proposed method is evaluated on PH2 Dermoscopy Image Dataset and Edinburgh Dermofit Image Library, with comparison against four alternative classification methods. Our method demonstrates the best performance, with $94.43 \%$ sensitivity and $81.01 \%$ specificity on PH2 dataset and $99.50 \%$ sensitivity and $93.68 \%$ specificity on Edinburgh Library.
\end{abstract}

\section{Introduction}

Skin cancer is the third most common human malignancy, being diagnosed mostly among light pigmented skin [7]. Skin cancer can be classified as melanoma and non-melanoma. Malignant melanoma causes the most mortality, although it is not the most prevalent among all skin cancer types [27]. Fortunately, an early screening of skin cancer can greatly increase the chance of cure. However, due to the similarity between nevi and early stage melanoma, as illustrated in Fig. 1, they are in many cases difficult to differentiate even for experienced skin specialists, not to mention for those non-experts and people with-

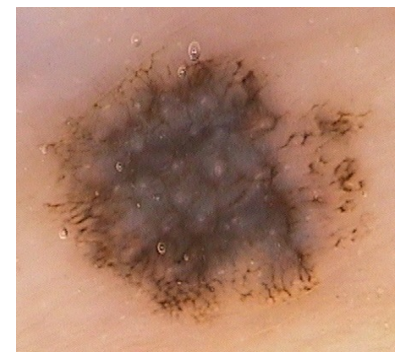

(a) Melanoma

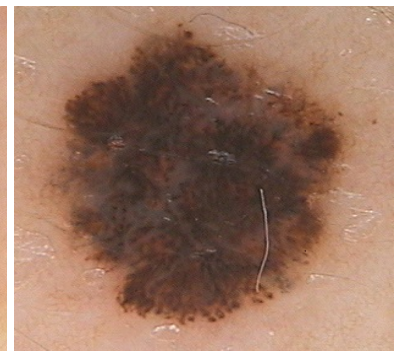

(b) Dysplastic Nevus
Figure 1: Illustration of melanoma and nevus

out medical background. As a consequence, diagnosis of melanoma is still heavily relied on biopsy.

To make a preliminary diagnosis, dermatologists usually use a non-invasive method, i.e., dermoscopy, to examine the suspected skin lesion before biopsy. However, this greatly depends on dermatologists' experience, turning out to be subjective and many times inaccurate. In order to tackle this problem, researchers are making efforts to build automatic image-based diagnosis systems. The development of melanoma detection methods in such systems is normally in accordance with the clinical rules which are based on the appearance of local certain colours or texture patterns, such as classical pattern analysis [22], ABCD rules [28], Menzies method [20] and 7-point checklist [2].

Most image-based skin cancer classifications methods follow a pipeline of pre-processing, segmentation, feature extraction and classification. Pre-processing aims at removing noises and occlusions as well as enhancing the focal areas in the image, after which feature extraction is conducted. Besides some commonly used feature extraction 
methods in computer vision, such as SIFT, LBP, GLCM, and color histogram etc., subtle features are extracted following clinical rules, such as streaks [25], the blue-whitish structures [5, 15] and pigmented network [26]. In more recent, deep learning is also applied to skin lesion extraction [9]. After feature extraction, images have to be encoded into vectorized representation for classification.

Normally, these feature vectors have a dimension of several hundreds. If they are classified directly, we cannot achieve the best classification performance, as recent studies show that dimensionality reduction can enhance the classification performance by projecting samples from highdimensional space to a low-dimensional space. However, seldom any work has been incorporated into the learning process of melanoma detection method. Therefore, this paper focuses on developing novel dimensionality reduction method for melanoma detection, in particular, via matrix factorization.

Commonly used matrix factorization methods include principle component analysis (PCA), singular value decomposition (SVD), QR decomposition (QRD), nonnegative matrix factorization (NMF), etc. Among these methods, NMF extracts part representation that can be used to identify semantic features in images. Given a vectorized image representation after feature extraction from each image, a feature matrix can be constructed from all data and then factorized into a basis matrix and a coefficient matrix. NMF has shown great flexibility of its framework [4, 13]. The iteration rules of NMF are simpler, easier to implement and faster to converge than other dimension reduction methods. Furthermore, NMF model has an explicit physical interpretation or explanation. Each column of the factorized basis matrix represents the cluster centre while the factorized coefficient matrix represents the closeness between each sample to its cluster centre, which benefits the incorporation of manifold of training data into testing data. However, most of NMF methods either fail to consider the intrinsic geometry of the data or are unsupervised methods without much discriminative ability.

To address this problem, Cai et al. [4] proposed an unsupervised manifold regularized method GNMF to enhance the clustering performance. The manifold regularizer takes the local interrelations between data points into account by constructing a nearest neighbour graph. GNMF can turn into a semi-supervised learning algorithm with GNMF weighting directly encoded into the graph structure. However, it might lead to over-fitting since it fails to consider the locally geometric structure of the data. To solve the over-fitting problem, instead of encoding the weight matrix directly, Ren et al. [24] proposes a method to involve Mahalanobis distance learning into GNMF so as to improve the clustering performance. Another solution is constrained NMF [13] which incorporates label information in the lower dimensional space by applying hard constraints on the coefficient matrix from the global perspective. However, this method fails to consider the local geometry of the data.

In this paper, we investigate both local manifold and global constraints in the data and propose a novel melanoma detection method based on Mahalanobis distance learning and constrained graph regularized nonnegative matrix factorization (MCNMF) so as to improve the classification performance. On one hand, a Mahalanobis distance matrix is learned from the training data, which later contributes to constructing the local manifold from the distribution of data points and image labels. On the other hand, the labels are applied as additional constraints to the coefficient matrix globally.

The rest of the paper is organized as follows. We introduce related NMF methods in the context of melanoma detection in section 2 . Section 3 describes the details of the proposed method. In order to verify the effectiveness of our method, experimental evaluations and comparison with other four commonly used classification methods are presented in Section 4. Finally, conclusions are drawn in Section 5.

\section{Related Work}

NMF aims at projecting a non-negative matrix into lower dimensional representations as the product of two matrices of non-negative elements. Given encoded feature matrix of all skin lesion images $\mathbf{X}=\left[\mathbf{x}_{1}, \ldots, \mathbf{x}_{m}\right] \in \mathbb{R}^{n \times m}$, two decomposed nonnegative matrices are basis matrix $\mathbf{U} \in \mathbb{R}^{n \times k}$ and its coefficient matrix $\mathbf{V} \in \mathbb{R}^{m \times k}$. Then the objective function of NMF is represented as:

$$
\arg \min _{U, V}\left\|\mathbf{X}-\mathbf{U V}^{T}\right\|_{F}^{2}, \quad \text { s.t. } \quad \mathbf{U} \geq 0, \mathbf{V} \geq 0
$$

On top of the objective function of NMF defined in Eq. (1), GNMF aims at building a graph for the data points in the new data space by considering the geometrical relations of points in the original space. This graph is used to regularize the NMF decomposition process, so as to generate consistent data distribution in the projected subspaces based on the manifold assumption.

Here, we consider the feature vector of each skin lesion as a data point or a vertex in a graph. The graph is built by firstly finding $N$ nearest neighbours for each data point and then building edges between the point and its neighbours. So $N$ nearest neighbors form $N$ vertices in the graph. Let the new basis of the low dimensional representation $\mathbf{V}$ as $\mathbf{v}_{j}=\left[\mathbf{v}_{j 1}, \ldots, \mathbf{v}_{j k}\right]^{T}$. The distance between two vertices $\mathbf{x}_{j}$ and $\mathbf{x}_{l}$ can be characterized using weight $\mathbf{w}_{j l}$, which is an entry in weight matrix $\mathbf{W}$. Therefore, we can use the 


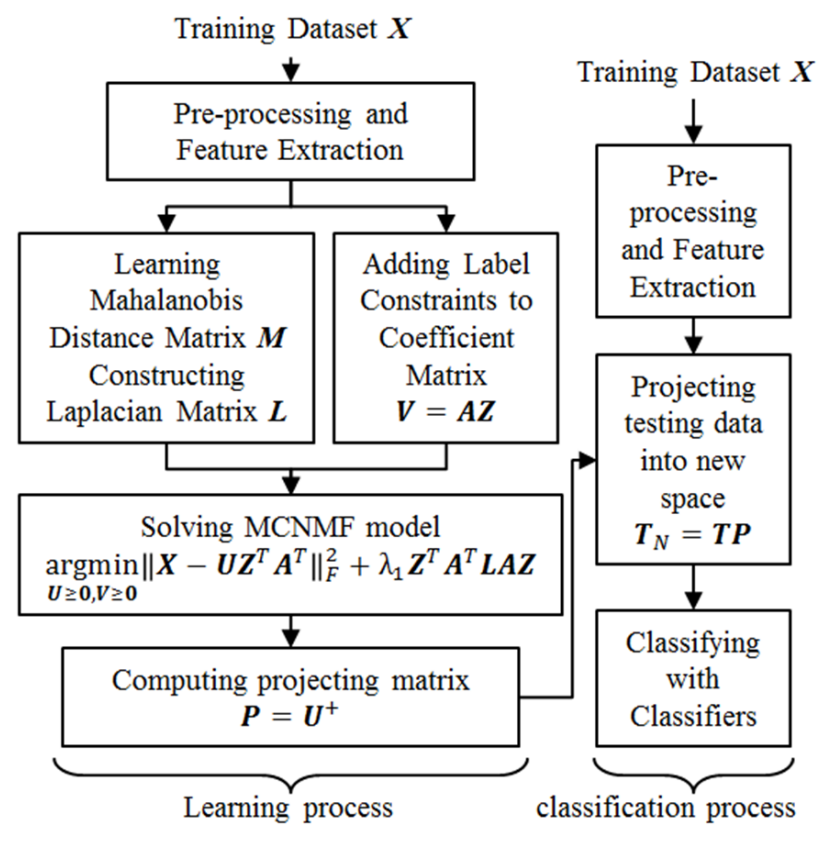

Figure 2: The proposed framework for dermoscopy image classification

following term to measure the smoothness of $\mathbf{V}$ :

$$
\begin{aligned}
\mathbf{R} & =\frac{1}{2} \sum_{j, l=1}^{N}\left\|\mathbf{x}_{j}-\mathbf{x}_{l}\right\|^{2} \mathbf{w}_{j l} \\
& =\operatorname{Tr}\left(\mathbf{V}^{T} \mathbf{D V}\right)-\operatorname{Tr}\left(\mathbf{V}^{T} \mathbf{W} \mathbf{V}\right) \\
& =\operatorname{Tr}\left(\mathbf{V}^{T} \mathbf{L V}\right)
\end{aligned}
$$

where $\operatorname{Tr}(\cdot)$ denotes the trace of a matrix and the entries of the diagonal matrix $\mathbf{D}$ equal to the column (or row, since $\mathbf{W}$ is symmetric) sum of $\mathbf{W}$, i.e., $\mathbf{D}_{j j}=\Sigma_{l} \mathbf{W}_{j l}$. $\mathbf{L} \triangleq \mathbf{D}-\mathbf{W}$ is a Laplacian matrix.

With the graph and Laplacian matrix constructed, we can use them as the manifold constraint for the GNMF model, so that the original geometrical relations of the skin lesion images can be embedded into the new space. It is expected that in the low dimensional space $\mathbf{v}_{j}$ is close to $\mathbf{v}_{l}$ if the $\mathbf{x}_{j}$ and $\mathbf{x}_{l}$ in the same class (either melanoma or nevus) are close. This can be achieved by solving the new objective function of NMF with manifold constraint. The objective function of GNMF is defined as follows

$$
\arg \min _{\mathbf{U}, \mathbf{V}}\left\|\mathbf{X}-\mathbf{U V}^{T}\right\|_{F}^{2}+\lambda \operatorname{Tr}\left(\mathbf{V}^{T} \mathbf{L V}\right)
$$

\section{Proposed Method}

In this section, we describe the proposed method based on Mahalanobis distance learning and constrained graph regularized NMF (MCNMF). As shown in Fig. 2, given a set of skin images, preprocessing and feature extraction are performed first. The feature extraction step also encodes the images into vectorized representations. To this end, we use several commonly used feature extraction methods in melanoma detection research, which will be described in Section 4 . The core procedure can then be divided into a learning stage and a classification stage. The learning stage uses label information in both local and global perspectives. First, we adopted a Mahalanobis distance learning method to enhance the discriminability of the proposed method from a local perspective. In the projected lowdimensional data, the learning method enlarges distances of data samples between different classes while reducing the distances among samples within the same class. Second, with the label constraints being added to the coefficient matrix, the discriminability can be enhanced from a global perspective, where the samples from the same class are projected to the same class in new lower dimensional space. After solving the objective function, we obtain a projecting matrix and go to the next stage. In the classification stage, the learned projection matrix is used to project the testing data into the same low dimensional space. At last, the new representation of the testing sample in the projected subspace is classified by a classifier.

In the following, we focus on the learning stage of our classification method and describe in detail the objective functions and their updating rules for obtaining the solutions. In addition, proof of the convergence of the objective function is also provided.

\subsection{Local Discriminative Analysis}

In GNMF model, it is the constructed weight matrix that matters. Instead of using fixed Euclidean distance as in NMF, in our method, the weight matrix is calculated through Mahalanobis distance learning [30], as distance metric learning is an important technique in introducing prior class knowledge into clustering or classification models [10]. The aim of this step is to effectively explore the label information on melanoma and nevus data so as to minimize the distances between similar samples in the same class while maximizing the distances between dissimilar samples in different classes. In addition, as we mentioned before, this can also avoid over-fitting by considering the locally geometric structure of data.

Here we borrow the data assumption in Section 2. Given a pair of data points $\mathbf{x}_{j}, \mathbf{x}_{l} \in \mathbb{R}^{n}$ describing the vectorized feature of two skin lesion images, their Mahalanobis distance $\mathbf{w}_{j, l} \in \mathbf{W}$ is calculated as follows

$$
\mathbf{w}_{j, l}=\sqrt{\left(\mathbf{x}_{j}-\mathbf{x}_{l}\right)^{T} \mathbf{M}\left(\mathbf{x}_{j}-\mathbf{x}_{l}\right)}
$$

where $\mathbf{M} \in \mathbb{R}^{n \times n}$ is positive semidefinite.

Instead of calculating the Mahalanobis distance matrix directly, we can obtain it through computing a simplified 
matrix beforehand to facilitate the calculation. This is achieved by using Cholesky decomposition. $\mathbf{M}$ can be decomposed into $\mathbf{M}=\mathbf{B B}^{T}$, where $\mathbf{B}$ is a lower triangular matrix, $\mathbf{B} \in \mathbb{R}^{n \times b}$ and $b \leqslant n$.

Here, we follow the work of Xiang et al [30] to learn the Mahalanobis distance matrix. First, the training dataset is divided into two groups: linking group $(\mathbb{L})$ and not-linking $(\mathbb{N})$ group. The linking group contains point pairs under the same class while the not-linking group contains points from different classes. Then matrix $\mathbf{B}$ can be learned from these two groups.

Denote the sum of squared distances between points within group $\mathbb{L}$ and $\mathbb{N}$ as $\mathbf{D}_{L}$ and $\mathbf{D}_{N}$ respectively.

$$
\begin{aligned}
\mathbf{D}_{L} & =\sum_{\left(\mathbf{x}_{j}, \mathbf{x}_{l}\right) \in \mathbb{L}}\left(\mathbf{B}^{T} \mathbf{x}_{j}-\mathbf{B}^{T} \mathbf{x}_{l}\right)^{T}\left(\mathbf{B}^{T} \mathbf{x}_{j}-\mathbf{B}^{T} \mathbf{x}_{l}\right) \\
& =\operatorname{tr}\left(\mathbf{B}^{T} \mathbf{S}_{L} \mathbf{B}\right) \\
\mathbf{D}_{N} & =\sum_{\left(\mathbf{x}_{j}, \mathbf{x}_{l}\right) \in \mathbb{N}}\left(\mathbf{B}^{T} \mathbf{x}_{j}-\mathbf{B}^{T} \mathbf{x}_{l}\right)^{T}\left(\mathbf{B}^{T} \mathbf{x}_{j}-\mathbf{B}^{T} \mathbf{x}_{l}\right) \\
& =\operatorname{tr}\left(\mathbf{B}^{T} \mathbf{S}_{N} \mathbf{B}\right)
\end{aligned}
$$

where $\mathbf{S}_{L}$ and $\mathbf{S}_{N}$ are the covariance matrices of the point pairs in $\mathbb{L}$ and $\mathbb{N}$ respectively, and are defined as

$$
\begin{aligned}
\mathbf{S}_{L} & =\sum_{\left(\mathbf{x}_{j}, \mathbf{x}_{l}\right) \in \mathbb{L}}\left(\mathbf{x}_{j}-\mathbf{x}_{l}\right)\left(\mathbf{x}_{j}-\mathbf{x}_{l}\right)^{T} \\
\mathbf{S}_{N} & =\sum_{\left(\mathbf{x}_{j}, \mathbf{x}_{l}\right) \in \mathbb{N}}\left(\mathbf{x}_{j}-\mathbf{x}_{l}\right)\left(\mathbf{x}_{j}-\mathbf{x}_{l}\right)^{T}
\end{aligned}
$$

In order to achieve the optimal $\mathbf{B}, \mathbf{D}_{N}$ shall be maximized and $\mathbf{D}_{L}$ shall be minimized. Therefore, we have the following objective function

$$
\min _{\mathbf{B}^{T} \mathbf{B}=\mathbf{I}} \frac{\operatorname{tr}\left(\mathbf{B}^{T} \mathbf{S}_{L} \mathbf{B}\right)}{\operatorname{tr}\left(\mathbf{B}^{T} \mathbf{S}_{N} \mathbf{B}\right)}
$$

Here, a constraint $\mathbf{B}^{T} \mathbf{B}=\mathbf{I}$ is introduced in order to avoid that the solutions to be degenerate [30], where $\mathbf{I} \in$ $\mathbb{R}^{b \times b}$. It shall be noted that if $b=n, \mathbf{B}^{T} \mathbf{B}=\mathbf{B B}^{T}=\mathbf{I}$, so that the distance will be Euclidean distance. Hence, we set condition $d<n$. Therefore, the Mahalanobis matrix $\mathbf{M}$ can be achieved as follows:

$$
\mathbf{M}=\mathbf{B}^{*}\left(\mathbf{B}^{*}\right)^{T}
$$

\subsection{Global Discriminative Analysis}

In addition to the local discriminative information introduced into the low-dimensional data, adding label information to global low-dimensional data improves discriminability. Here, we treat labels of the data samples as hard constraints to preserve the labels in the new low-dimensional space.
Assume there are $p$ melanoma samples in $\mathbf{X}$, while the other $m-p$ samples are nevi. We label melanoma as 1 , and nevi as 2 . If a sample $x_{i}$ belongs to class $j \in\{1,2\}$, then we denote it as $c_{i j}=1$. Otherwise, $c_{i j}=0$. The constraint matrix is defined as:

$$
\mathbf{A}=\left[\begin{array}{l}
\mathbf{C}_{p 1} \\
\mathbf{C}_{p 2}
\end{array}\right]=\left[\begin{array}{cc}
1 & 0 \\
\cdots & \ldots \\
1 & 0 \\
0 & 1 \\
\cdots & \ldots \\
0 & 1
\end{array}\right]
$$

where $\mathbf{C}_{p 1} \in \mathbb{R}^{p \times 2}$ is a matrix with 1 in the first column and 0 in the second column, while $\mathbf{C}_{p 2} \in \mathbb{R}^{(m-p) \times 2}$ is a matrix with 0 in the first column and 1 in the second column.

Then, we use this constraint matrix to map the label information into the new low-dimensional data, so as to label the coefficient matrix. Hence, after introducing an auxiliary matrix $\mathbf{Z}$, the mapping can be denoted as:

$$
\mathbf{V}=\mathbf{A} \mathbf{Z}
$$

where, $\mathbf{Z} \in \mathbb{R}^{2 \times k}, k \ll m$ and $k \ll n$.

\subsection{Objective Function}

By imposing the global constraints and local manifold into GNMF, our proposed objective function is:

$$
\arg \min _{\mathbf{U}, \mathbf{Z}}\left\|\mathbf{X}-\mathbf{U} \mathbf{Z}^{T} \mathbf{A}^{T}\right\|_{F}^{2}+\lambda \operatorname{Tr}\left(\mathbf{Z}^{T} \mathbf{A}^{T} \mathbf{L A Z}\right)
$$

where the Laplacian matrix $\mathbf{L} \triangleq \mathbf{D}-\mathbf{W}$, after introducing the matrix $\mathbf{M}$, the entries of $\mathbf{W}$ are defined in Eq. 4, $\mathbf{M}=\mathbf{A} \mathbf{A}^{T}$, and $\mathbf{A}$ is learned from the Mahalanobis distance learning.

\subsection{Updating Rules}

We now discuss how to achieve the optimal weight matrix $\mathbf{A}$ and lower dimensional matrices $\mathbf{U}, \mathbf{Z}$ through minimizing the two objective functions defined in Eqs. (9) and (12).

We start from solving the objective function in Eq. (9), where two cases are considered according to whether the denominator $\operatorname{tr}\left(\mathbf{A}^{T} \mathbf{S}_{N} \mathbf{A}\right)$ is zero or not. This determines the relationship between $b$ and $n-r$, where $r$ is the rank of the matrix $\mathbf{S}_{N}$. Details on two cases and the complete updating algorithm can be found in [30].

After obtaining the Mahalanobis distance matrix, we impose it to Eq. (12) and solve the minimization problem in Eq. (12). We obtain the local minima by iteratively updating two rules regarding $\mathbf{U}$ and $\mathbf{Z}$ respectively, since it is obvious that Eq. (12) is not convex if both $\mathbf{U}$ and $\mathbf{Z}$ are changing together.

Here we introduce the Lagrange multipliers, $\psi_{i k}$ and $\phi_{j k}$ for $\psi_{i k} \geq 0, \phi_{j k} \geq 0$ and $\Psi=\left[\psi_{i k}\right], \Phi=\left[\phi_{j k}\right]$. Before 


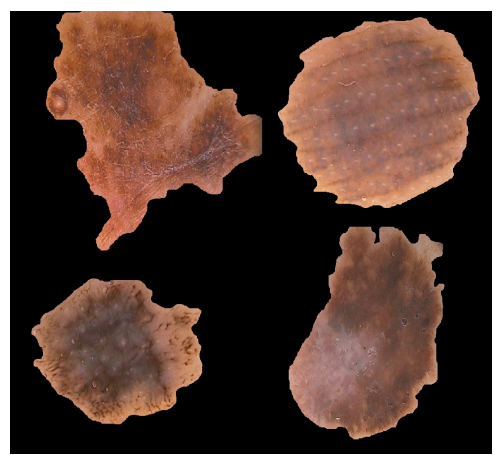

(a) Melanoma on PH2 dataset

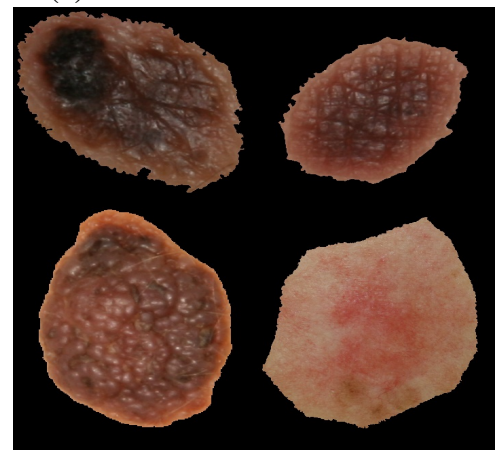

(c) Melanoma on Edinburgh dataset

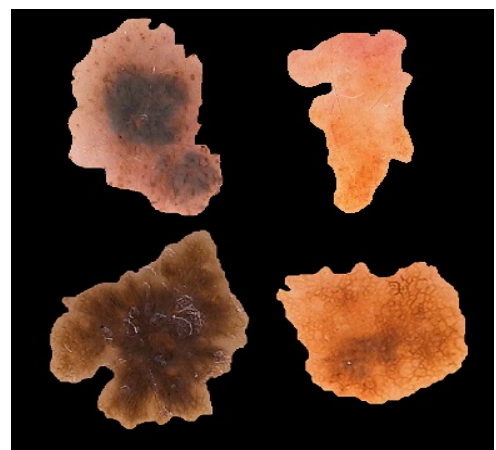

(b) Nevus on PH2 dataset

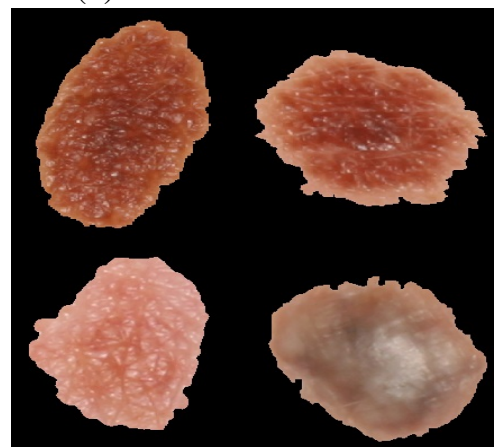

(d) Nevus on Edinburgh dataset

Figure 3: Sample images on PH2 and Edinburgh datasets.

that, the term in Eq. (12) to be optimized can be rewritten as:

$$
\begin{aligned}
O= & \left\|\mathbf{X}-\mathbf{U} \mathbf{Z}^{T} \mathbf{A}^{T}\right\|_{F}^{2}+\lambda \operatorname{Tr}\left(\mathbf{Z}^{T} \mathbf{A}^{T} \mathbf{L A Z}\right) \\
= & \operatorname{Tr}\left(\mathbf{X}-\mathbf{U} \mathbf{Z}^{T} \mathbf{A}^{T}\right)\left(\mathbf{X}-\mathbf{U} \mathbf{Z}^{T} \mathbf{A}^{T}\right)+ \\
& \lambda \operatorname{Tr}\left(\mathbf{Z}^{T} \mathbf{A}^{T} \mathbf{L} \mathbf{A Z}\right) \\
= & \operatorname{Tr}\left(\mathbf{X} \mathbf{X}^{T}\right)-2 \operatorname{Tr}\left(\mathbf{X} \mathbf{A Z} \mathbf{U}^{T}\right)+ \\
& \operatorname{Tr}\left(\mathbf{U} \mathbf{Z}^{T} \mathbf{A}^{T} \mathbf{A} \mathbf{Z} \mathbf{U}^{T}\right)+\lambda \operatorname{Tr}\left(\mathbf{Z}^{T} \mathbf{A}^{T} \mathbf{L A Z}\right)(13)
\end{aligned}
$$

Then, the Lagrange $\mathcal{L}$ is:

$$
\mathcal{L}=O+\operatorname{Tr}\left(\Psi \mathbf{U}^{T}\right)+\operatorname{Tr}\left(\Phi \mathbf{Z}^{T}\right)
$$

In order to achieve the local minima, we calculate the partial derivatives of $\mathcal{L}$ regarding to $\mathbf{U}$ and $\mathbf{Z}$ respectively, thereby having:

$$
\begin{gathered}
\frac{\partial \mathcal{L}}{\partial \mathbf{U}}=-2 \mathbf{X} \mathbf{A} \mathbf{Z}+2 \mathbf{U} \mathbf{Z}^{T} \mathbf{A}^{T} \mathbf{A} \mathbf{Z}+\Psi \\
\frac{\partial \mathcal{L}}{\partial \mathbf{Z}}=-2 \mathbf{A}^{T} \mathbf{X}^{T} \mathbf{U}+2 \mathbf{A}^{T} \mathbf{A} \mathbf{Z} \mathbf{U}^{T} \mathbf{U}+2 \lambda \mathbf{A}^{T} \mathbf{L A Z}+\Phi
\end{gathered}
$$

Due to KKT conditions $\psi_{i k} \mathbf{u}_{i k}=0, \phi_{j k} \mathbf{z}_{j k}=0$, we have the final updating rules for $\mathbf{u}_{i k}$ and $\mathbf{z}_{j k}$ :

$$
\mathbf{u}_{i k}^{t+1} \leftarrow \mathbf{u}_{i k}^{t} \frac{(\mathbf{X} \mathbf{A} \mathbf{Z})_{i k}}{\left(\mathbf{U} \mathbf{Z}^{T} \mathbf{A}^{T} \mathbf{A} \mathbf{Z}\right)_{i k}}
$$

$$
\mathbf{z}_{j k}^{t+1} \leftarrow \mathbf{z}_{j k}^{t} \frac{\left(\mathbf{A}^{T} \mathbf{X}^{T} \mathbf{U}+\lambda \mathbf{A}^{T} \mathbf{W A Z}\right)_{j k}}{\left(\mathbf{A}^{T} \mathbf{A} \mathbf{Z} \mathbf{U}^{T} \mathbf{U}+\lambda \mathbf{A}^{T} \mathbf{D A Z}\right)_{j k}}
$$

Regarding to the updating rules above, the objective function (12) is non-increasing. The objective function is invariant under these updates if and only if $\mathbf{U}$ and $\mathbf{Z}$ are at a stationary point. As we only impose the learned distance matrix into the graph, the proof of convergence is then similar to the proof in GNMF [4] and CNMF [13] and is omitted due to the space limitation.

\section{Experiments and Results}

\subsection{Dataset}

We evaluated the proposed method on two datasets, $\mathrm{PH} 2$ Dermoscopy Image Dataset [19] and Edinburgh Dermofit Image Library [1]. Both datasets provide binary masks on skin lesion regions as well as skin cancer labels. PH2 is a relatively small dataset with 200 dermoscopy images, including 40 melanoma images and 160 nevus images. The Edinburgh library contains rather comprehensive classes of images. Besides 76 melanoma images, it has skin images in 9 classes, including 331 dysplastic nevus images. In the experiments, we used all melanoma images and the same number of randomly selected dysplastic nevus images. 


\subsection{Experimental Setting}

We randomly took half of the images in each class as the training set while using the other half as the testing set. We ran the experiments for ten times and calculated means and standard deviations as the final results. It should be noted that since the performance of NMF methods is greatly impacted by the initialization condition, we repeated the GNMF methods for 200 times and adopted the results with the best sensitivity. Before extracting features, all the images were segmented with the binary masks and were preprocessed with the DullRazor hair removal algorithm [12]. Some samples segmented and pre-processed images in two datasets are shown in Fig. 3.

To demonstrate how the proposed method performs on different feature extraction methods, we tested our method on four features, namely, SIFT [14], Local Binary Patterns (LBP) [8], Colour histogram (ClrHst) and Gray-Level CoOccurrence Matrix (GLCM) [21], which are all commonly used features in melanoma classification research $[23,11$, $16,17]$. The SIFT feature was used with the Bag of Words method to generate vectorized image representation with a dimension of 200. Instead of analyzing each single pattern and extracting features for each specific pattern, bag of words allows the skin lesion be effectively represented as the distribution of the most distinct local features. Local binary patterns assigns a binary label to every center pixel of an image by thresholding its $3 \times 3$ neighbourhood, so each point can be represented by a 8 -digit number. Then a 256-dimensional vector can be generated, which records the histogram of each combination of whether the center is greater or less than the neighbourhood. Colour histogram is a widely used colour feature descriptor in melanoma diagnosis. This descriptor is a 144-dimension vector, including the histogram of the nine channels in three colour space, i.e., RGB, HSV, and LAB. GLCM is a classic texture descriptor and has been widely used to describe dermoscopy images. The texture features are extracted based on statistics measurement of co-occurrence probabilities between two pixels. It calculates how often a pixel with gray scale intensity occurs adjacent to a pixel with another value. The spatial distance and orientation between the adjacent point pairs are related to the distance and the direction. Here, we set the size of LBP vector as 256.

To show the effectiveness of our proposed dimensionality reduction method, after MCNMF was applied, we used support vector machine (SVM), and random forest (RF) to classify the projected data. These two classifiers are commonly used for melanoma detection $[18,23,29]$. We also present the results of SVM and RF with the raw features. For the SVM classifier, we used LIBSVM [6] to implement the training and testing step. When random Forest [3] was used, we adopted a two-stage classification implementation. First, 20 decision trees were trained from the original data.
Then the method was trained by bootstrap aggregating and the best split among the randomly selected subset of features. In the testing step, a sample was labelled by majority voting. In addition, a related NMF method, constrained non-negative factorization (CNMF), was used for comparison.

There are two important parameters in the proposed method, regularization parameter $\lambda$ and the number of nearest neighbors in the constructed manifold $p$. $\lambda$ controls the contribution of the data reconstruction term and the manifold regularization term. Since the vectorized data representation are constructed from different features, this leads to varied influence of regularization term. On the other hand, similar to GNMF, $p$ has a great impact on the local geometric structure of the data distribution. Hence, we ran experiments with $\lambda \in\left[10^{-3}, 10^{3}\right]$ and $p \in[3,21]$ respectively on the mean sensitivity of ten groups of randomly sampled data on both datasets.

We used sensitivity (SE) and specificity (SP), which are two commonly adopted criteria in melanoma diagnosis, to evaluate various methods. Sensitivity measures the proportion of melanoma images that are correctly identified, i.e., the number of correctly classified melanoma images divided by the sum of the number of correctly classified melanoma images and the number of falsely classified nevus images. Specificity measures the proportion of negatives that are correctly identified, i.e., the number of correctly classified nevus images divided by the sum of the number of correctly classified nevus images and the number of falsely classified melanoma images. For both criteria, a higher value implies a better performance. Moreover, in melanoma detection, higher sensitivity is preferred over specificity, as correctly detected melanoma is more important. Hence, when tuning the parameters, we chose the ones that generate the best performance in terms of sensitivity.

\subsection{Results and Analysis}

In the beginning, we tune the parameter $\lambda$ and $p$ so as to achieve the best performance of the MCNMF method. Fig. (4) illustrates the influence of $\lambda$ on the sensitivity of all the four features on both datasets. Except LBP, sensitivity of all other features remains stable when $\lambda$ varies, while sensitivity of LBP drops when $\lambda$ increases on PH2 and peaks around $\lambda=1$ on Edinburgh. Therefore, we set $\lambda$ according to the maximum performance for each feature on either dataset. Fig. (5) shows the influence of $p$ on the sensitivity of all the four features on both datasets, yet we set the value of $p$ for each feature according to the best experimental results. It was reported that the performance tends to fade when $p$ increases [4]. However, in our result, this tendency is quite subtle. LBP increases when $p>17$ on PH2, and $p>8$ on Edinburgh. This may be due to the influence of the global constraint. When $p$ increases, the impact of local 
Table 1: Experimental results

\begin{tabular}{|c|c|c|c|c|c|c|c|c|c|c|}
\hline \multirow{2}{*}{ Methods } & \multirow{2}{*}{\multicolumn{2}{|c|}{$\begin{array}{l}\text { Dataset } \\
\text { Features }\end{array}$}} & \multicolumn{4}{|c|}{ PH2 } & \multicolumn{4}{|c|}{ Edinburgh } \\
\hline & & & LBP & SIFT & ClrHst & GLCM & LBP & SIFT & ClrHst & GLCM \\
\hline \multirow{4}{*}{$\begin{array}{c}\text { MCNMF } \\
+ \text { SVM }\end{array}$} & \multirow{2}{*}{$\mathrm{SE} \%$} & MNS & 94.43 & 87.80 & 91.33 & 90.67 & 98.95 & 99.20 & 97.64 & 99.22 \\
\hline & & STD & 3.32 & 4.26 & 4.21 & 4.53 & 1.36 & 1.28 & 2.83 & 1.74 \\
\hline & \multirow{2}{*}{$\mathrm{SP} \%$} & MNS & 81.01 & 90.94 & 80.07 & 78.63 & 96.23 & 93.28 & 93.12 & 93.38 \\
\hline & & STD & 8.98 & 6.11 & 7.10 & 9.68 & 2.35 & 4.16 & 3.08 & 5.14 \\
\hline \multirow{4}{*}{$\begin{array}{c}\text { MCNMF } \\
+ \text { RF }\end{array}$} & \multirow{2}{*}{$\mathrm{SE} \%$} & MNS & 94.27 & 89.28 & 92.31 & 92.27 & 97.64 & 99.50 & 96.94 & 96.63 \\
\hline & & STD & 4.55 & 4.13 & 6.32 & 5.57 & 3.29 & 1.58 & 3.62 & 3.83 \\
\hline & \multirow{2}{*}{$\mathrm{SP} \%$} & MNS & 82.07 & 78.98 & 82.39 & 83.92 & 94.02 & 93.68 & 89.84 & 92.47 \\
\hline & & STD & 10.62 & 8.54 & 10.16 & 10.49 & 7.63 & 4.93 & 8.61 & 8.83 \\
\hline \multirow{4}{*}{$\begin{array}{l}\text { CNMF } \\
+ \text { SVM }\end{array}$} & \multirow{2}{*}{ SE\% } & MNS & 86.40 & 84.96 & 87.15 & 87.51 & 93.46 & 94.50 & 90.16 & 87.26 \\
\hline & & STD & 4.69 & 5.11 & 4.21 & 4.34 & 2.99 & 2.93 & 2.76 & 2.73 \\
\hline & \multirow{2}{*}{$\mathrm{SP} \%$} & MNS & 80.86 & 73.48 & 79.58 & 77.53 & 96.50 & 97.83 & 97.76 & 98.23 \\
\hline & & STD & 8.76 & 6.15 & 8.26 & 9.05 & 2.51 & 1.67 & 2.16 & 1.53 \\
\hline \multirow{4}{*}{$\begin{array}{c}\text { CNMF } \\
+\mathrm{RF}\end{array}$} & \multirow{2}{*}{$\mathrm{SE} \%$} & MNS & 74.51 & 76.69 & 74.28 & 76.88 & 92.56 & 94.62 & 91.05 & 90.08 \\
\hline & & STD & 9.00 & 11.75 & 6.06 & 7.28 & 4.01 & 4.00 & 4.59 & 2.96 \\
\hline & \multirow{2}{*}{$\mathrm{SP} \%$} & MNS & 72.91 & 72.32 & 74.19 & 77.30 & 91.95 & 94.64 & 92.71 & 94.04 \\
\hline & & STD & 7.30 & 6.62 & 8.79 & 9.41 & 4.60 & 4.19 & 3.43 & 5.12 \\
\hline \multirow{4}{*}{ SVM } & \multirow{2}{*}{ SE\% } & MNS & 86.19 & 86.39 & 86.59 & 87.71 & 93.21 & 93.59 & 92.96 & 89.38 \\
\hline & & STD & 4.43 & 5.43 & 4.62 & 4.32 & 2.99 & 2.66 & 2.66 & 4.21 \\
\hline & \multirow{2}{*}{$\mathrm{SP} \%$} & MNS & 80.86 & 72.27 & 79.44 & 77.07 & 96.48 & 98.41 & 96.26 & 96.98 \\
\hline & & STD & 8.99 & 5.52 & 8.32 & 8.61 & 2.55 & 2.23 & 3.09 & 2.57 \\
\hline \multirow{4}{*}{$\mathrm{RF}$} & \multirow{2}{*}{ SE\% } & MNS & 82.60 & 77.21 & 84.99 & 85.91 & 93.89 & 95.37 & 96.35 & 96.64 \\
\hline & & STD & 8.91 & 5.96 & 7.84 & 6.91 & 4.07 & 2.80 & 2.45 & 3.14 \\
\hline & \multirow{2}{*}{$\mathrm{SP} \%$} & MNS & 75.92 & 77.32 & 83.10 & 79.68 & 92.96 & 96.56 & 95.46 & 95.19 \\
\hline & & STD & 7.72 & 11.55 & 9.35 & 7.65 & 3.87 & 2.45 & 3.64 & 3.04 \\
\hline
\end{tabular}

manifold drops, and hence the global constraints has more influence on the performance.

We present the melanoma detection results in Table 1. It can be observed that the proposed MCNMF method has achieved the best results on both datasets, which are $94.43 \%$ (SE) and $81.01 \%$ (SP) with LBP and SVM on PH2, and $99.5 \%$ (SE) and $93.68 \%$ (SP) with SIFT features and RF on the Edinburgh library. Since we consider SE as a more important evaluation criteria, when choosing all the parameters to achieve the best SE, our MCNMF method has significantly outperformed all other approaches, no matter which feature is used. Although applying SVM directly on feature vectors on Edinburgh show promising specificity, it sacrifices sensitivity. The effectiveness of the dimensionality reduction method can also be illustrated by the comparison with SVM and RF with raw features. Using classifiers directly is inferior to adopting dimensionality reduction with either MCNMF or CNMF before the classification step. Furthermore, we can also see that our method outperforms CNMF with the same SVM and RF classifier. This is because the local manifold that we build improves the discriminability. The standard deviations of most results from the MCNMF method are relatively small compared with those of most other methods, showing that our method is more robust in dealing with random split of training and testing images.

Moreover, datasets themselves also have great influence on the classification results. Table 1 shows that RF generally outperforms SVM on PH2, but SVM performs better than RF on Edinburgh, under the comparison of each single feature. That is mainly due to the difference of quality of the images and the image capturing conditions between these two datasets. In general, our proposed method achieves the best performance on both datasets compared with other classification methods.

Among all the features, LBP, color histogram, and GLCM are quite promising on $\mathrm{PH} 2$ while all features seem suitable for classifying images from Edinburgh dataset. LBP, SIFT and GLCM have achieved similar results on Edinburgh, indicating that texture features might be more effective than color distribution to distinguish Melanoma and Nevus. On PH2 dataset, color histogram shows good performance as well as texture features, which means color feature is also valid for feature extraction. 


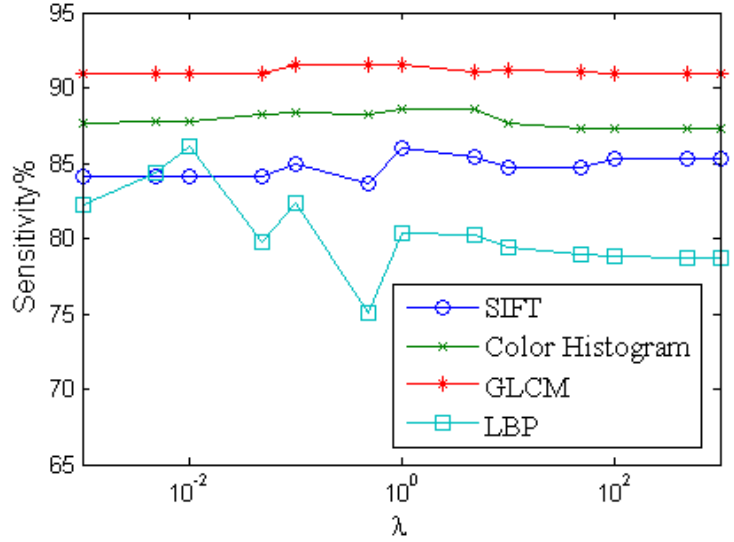

(a) Sensitivity on PH2 dataset

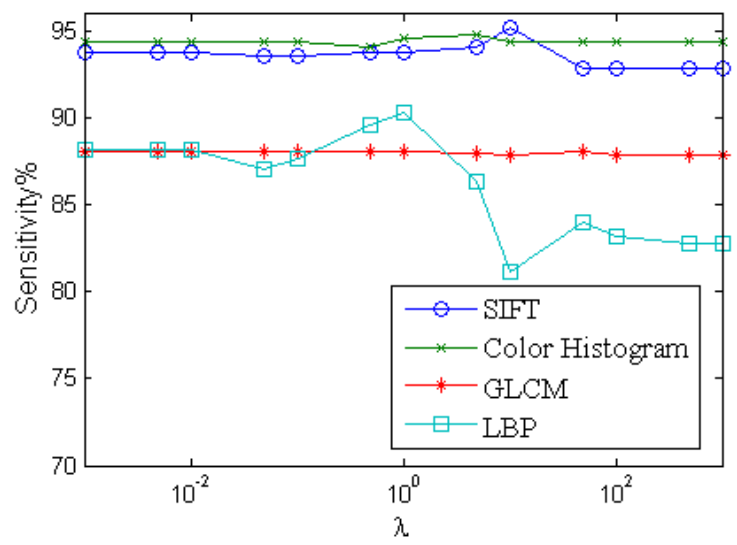

(b) Sensitivity on Edinburgh dataset

Figure 4: Sensitivity of MCNMF when parameter $\lambda$ changes with different features extracted on PH2 and Edinburgh dataset.

\section{Conclusions}

In this paper, we have introduced a method to classify melanoma from dysplastic nevi. We put our emphasis on dimensionality reduction before classification and proposed a novel classification method based on Mahalanobis distance learning and constrained graph regularized nonnegative matrix factorization. This makes it possible to involve the geometric information of training data into the projection of testing data, so as to enlarge the distances between classes and improve the classification results. The proposed method has been evaluated on two datasets with four features and compared with four alternative classification methods. Our method has achieved the best results in terms of sensitivity. In the current stage, our work uses only single feature to represent the image data. In the fu-

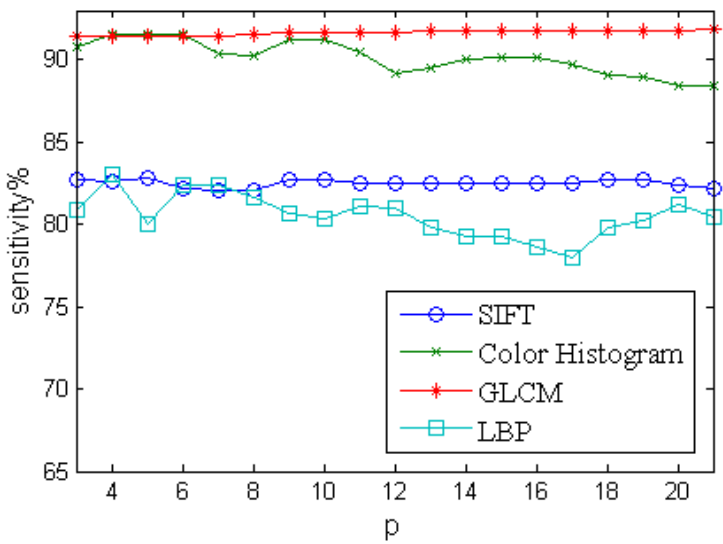

(a) Sensitivity on $\mathrm{PH} 2$ dataset

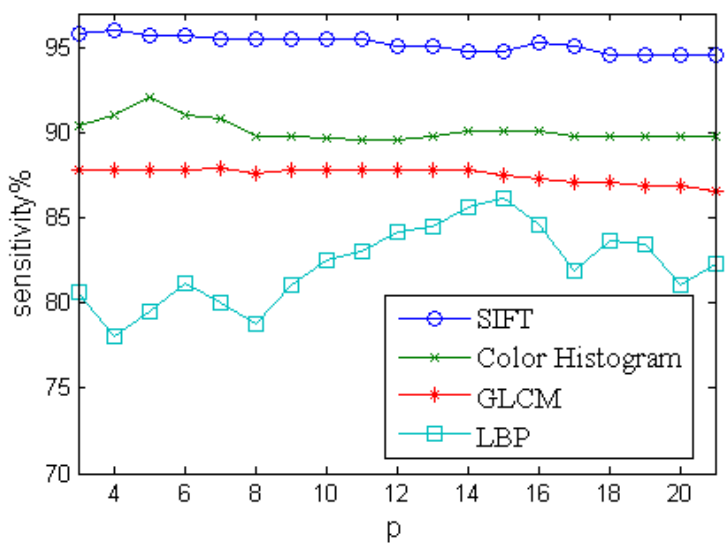

(b) Sensitivity on Edinburgh dataset

Figure 5: The sensitivity of MCNMF when parameter $p$ changes with different features extracted on $\mathrm{PH} 2$ and Edinburgh dataset.

ture work, we will look into the fusion of multiple features on existing data and exploring deep learning methods when larger amount of images are available.

\section{References}

[1] Dermofit image library. https:// licensing.eri.ed.ac.uk/i/software/ dermofit-image-library.html, 2016.

[2] G. Argenziano, G. Fabbrocini, P. Carli, V. D. Giorgi, E. Sammarco, and M. Delfino. Epiluminescence microscopy for the diagnosis of doubtful melanocytic skin lesions: comparison of the ABCD rule of dermatoscopy and a new 7-point checklist based on pattern analysis. Archives of Dermatology, 134(12):1563-1570, 1998.

[3] L. Breiman. Random forests. Machine Learning, 45(1):532, 2001. 
[4] D. Cai, X. He, J. Han, and T. Huang. Graph regularized nonnegative matrix factorization for data representation. IEEE Transactions on Pattern Analysis and Machine Intelligence, 33(8):1548-1560, 2011.

[5] M. E. Celebi, H. Iyatomi, W. V. Stoecker, R. H. Moss, H. S. Rabinovitz, G. Argenziano, and H. P. Soyer. Automatic detection of blue-white veil and related structures in dermoscopy images. Computerized Medical Imaging and Graphics, 32(8):670-677, 2008.

[6] C.-C. Chang and C.-J. Lin. LIBSVM: a library for support vector machines. ACM Transactions on Intelligent Systems and Technology, 2(27):1-27, 2011.

[7] V. Gray-Schopfer, C. Wellbrock, and R. Marais. Melanoma biology and new targeted therapy. Nature, 445(7130):851857, 2007.

[8] R. M. Haralick, K. Shanmugam, and H. Dinstein. Textural features for image classification. IEEE Transactions on Pattern Analysis and Machine Intelligence, 24(7):971-987, 2002.

[9] M. H. Jafari, E. Nasr-Esfahani, N. Karimi, S. M. Soroushmehr, S. Samavi, and K. Najarian. Extraction of skin lesions from non-dermoscopic images using deep learning. 2016.

[10] M. Koestinger, M. Hirzer, P. Wohlhart, P. M. Roth, and H. Bischof. Large scale metric learning from equivalence constraints. In CVPR, pages 2288-2295, 2012.

[11] K. Korotkov and R. Garcia. Computerized analysis of pigmented skin lesions: a review. Artificial Intelligence in Medicine, 56(2):69-90, 2012.

[12] T. Lee, V. Ng, R. Gallagher, A. Coldman, and D. McLean. Dullrazor $\cap$ : a software approach to hair removal from images. Computers in Biology and Medicine, 27(6):533-543, 1997.

[13] H. Liu, Z. Wu, D. Cai, and T. S. Huang. Constrained nonnegative matrix factorization for image representation. IEEE Transactions on Pattern Analysis and Machine Intelligence, 34(7):1299-1311, 2012.

[14] D. G. Lowe. Object recognition from local scale-invariant features. In The proceedings of the seventh IEEE International Conference on Computer Vision, volume 2, pages 1150 - 1157, 1999.

[15] A. Madooei, M. S. Drew, M. Sadeghi, and M. S. Atkins. Automatic detection of blue-white veil by discrete colour matching in dermoscopy images. In MICCAI, pages 453460. Springer, 2013.

[16] I. Maglogiannis and C. N. Doukas. Overview of advanced computer vision systems for skin lesions characterization. IEEE Transactions on Information Technology in Biomedicine, 13(5):7213-733, 2009.

[17] A. Masood and A. A. Al-Jumaily. Computer aided diagnostic support system for skin cancer: a review of techniques and algorithms. International Journal of Biomedical Imaging, pages 1-22, 2013.

[18] A. Masood, A. A. Al-Jumaily, and K. Anam. Self-supervised learning model for skin cancer diagnosis. In 7th International IEEE/EMBS Conference on Neural Engineering, pages 1012-1015, 2015.
[19] T. Mendonca, P. M. Ferreira, J. S. Marques, A. R. Marcal, and J. Rozeira. PH2 - a dermoscopic image database for research and benchmarking. In $E M B C$, pages 5437-5440, 2013.

[20] S. W. Menzies, C. Ingvar, K. A. Crotty, and W. H. McCarthy. Frequency and morphologic characteristics of invasive melanomas lacking specific surface microscopic features. Archives of Dermatology, 132(10):1178-1182, 1996.

[21] T. Ojala, M. Pietikäinen, and T. Maenpaa. Multiresolution gray-scale and rotation invariant texture classification with local binary patterns. IEEE Transactions on Systems, Man and Cybernetics, 6:610-621, 1973.

[22] H. Pehamberger, A. Steiner, and K. Wolff. In vivo epiluminescence microscopy of pigmented skin lesions. I. pattern analysis of pigmented skin lesions. Journal of the American Academy of Dermatology, 17(4):571-583, 1987.

[23] M. Rastgoo, R. Garcia, O. Morel, and F. Marzani. Automatic differentiation of melanoma from dysplastic nevi. Computerized Medical Imaging and Graphics, 43:44-52, 2015.

[24] W. Ren, G. Li, D. Tu, and L. Jia. Nonnegative matrix factorization with regularizations. IEEE Journal on Emerging and Selected Topics in Circuits and Systems, 4(1):153-164, 2014.

[25] M. Sadeghi, T. K. Lee, D. McLean, H. Lui, and M. S. Atkins. Detection and analysis of irregular streaks in dermoscopic images of skin lesions. IEEE Transactions on Medical Imaging, 32(5):849-861, 2013.

[26] M. Sadeghi, M. Razmara, T. K. Lee, and M. S. Atkins. A novel method for detection of pigment network in dermoscopic images using graphs. Computerized Medical Imaging and Graphics, 35(2):137-143, 2011.

[27] R. L. Siegel, K. D. Miller, and A. Jemal. Cancer statistics, 2015. A Cancer Journal for Clinicians, 65(1):5-29, 2015.

[28] W. Stolz, A. Riemann, A. B. Cognetta, L. Pillet, W. Abmayr, D. Holzel, P. Bilek, F. Nachbar, and M. Landthaler. ABCD rule of dermatoscopy - a new practical method for early recognition of malignant melanoma. European Journal of Dermatology, 4(7):517-527, 1994.

[29] E. L. Torre, B. Caputo, and T. Tommasi. Learning methods for melanoma recognition. International Journal of Imaging Systems and Technology, 20(4):316-322, 2010.

[30] S. Xiang, F. Nie, and C. Zhang. Learning a Mahalanobis distance metric for data clustering and classification. Pattern Recognition, 41(12):3600-3612, 2008. 